November 15, 2018

\title{
A New "Null-Plane" Quantum Poincaré Algebra
}

\author{
A. Ballesteros ${ }^{1}$, F.J. Herranz ${ }^{1}$, M.A. del Olmo ${ }^{2}$ and M. Santander ${ }^{2}$ \\ 1 Departamento de Física Aplicada, Universidad de Burgos \\ E-09003, Burgos, Spain \\ 2 Departamento de Física Teórica, Universidad de Valladolid \\ E-47011, Valladolid, Spain
}

\begin{abstract}
A new quantum deformation, which we call null-plane, of the $(3+1)$ Poincaré algebra is obtained. The algebraic properties of the classical null-plane description are generalized to this quantum deformation. In particular, the classical isotopy subalgebra of the null-plane is deformed into a Hopf subalgebra, and deformed spin operators having classical commutation rules can be defined. Quantum Hamiltonian, mass and position operators are studied, and the null-plane evolution is expressed in terms of a deformed Schrödinger equation.
\end{abstract}




\section{Introduction}

A central problem of a Hamiltonian formulation of quantum relativistic systems consists in the obtention of a set of dinamically independent variables in terms of the generators of the $(3+1)$ Poincaré group. For the null-plane evolution [1] this has been thorougly studied, and in this case it is well known that the information provided by the Poincaré invariance into a kinematical and a dynamical part. To achieve this by preserving causality, one can consider that the initial state of the system is defined on a light-like plane $\Pi_{n}^{\tau}$ (a hypersurface of points $x$ of the Minkowski space such that $n \cdot x=\tau$, where $n$ is a light-like vector and $\tau$ a real constant). The stability group $S_{+}$of $\Pi_{n}^{\tau}$ gives the kinematics inside the null-plane, and the dynamics is obtained by means of the remaining generators (the Hamiltonians) that map $\Pi_{n}^{\tau}$ into some other surface and describe the evolution of the system. A brief summary

of this construction is given in Section 2 for the case $n=\left(\frac{1}{2}, 0,0, \frac{1}{2}\right)$ (see [2] for a general review).

In this letter we present a new quantum deformation of the Poincaré algebra such that the classical null-plane isotopy subalgebra $S_{+}$is promoted into a Hopf subalgebra under deformation. The classical null-plane separation between kinematics and dynamics is therefore preserved in the quantum case. So, the study of deformations of the classical models built within this framework (for instance, the infinite momentum frame approach [3], gauge field theory quantized on null-planes [4] and applications in Hadron spectroscopy [5, 6]) could be physically interesting.

We also recall that previous Hopf algebra deformations of the $(3+1)$ Poincaré algebra [7, 8, 9] have been obtained within the purely kinematical framework where their classical counterparts live. However, the quantum algebra here introduced induces a (dynamical) deformation of the Schrödinger equation governing the evolution of the wave functions with support on the null-plane.

From an algebraic point of view the $(3+1)$ Poincaré deformation introduced in Section 3 is a quantization of a coboundary Lie bialgebra generated by a (nonstandard) $r$-matrix that fulfills the classical Yang-Baxter equation (CYBE). Starting from a similar $(2+1)$ null-plane deformation, which was introduced in [10], this new $(3+1)$ quantum Poincaré algebra is obtained by applying a deformation embedding method (fully described in [9, 11] for the standard deformation). It is worth remarking that the deformation of the most relevant structures in the classical nullplane description is straightforward: a centrally extended $(2+1)$ Galilean subalgebra of $\mathcal{P}(3+1)$ is transformed into a quantum algebra under deformation, the quantum central elements can be used to define the deformed mass and Pauli-Lubanski operators, and besides the latter induces a natural definition for the quantum spin operators, whose commutation rules are shown to be the classical ones. Quantum analogues of reduced Hamiltonians, position operators and the deformed uncertainty relations linked to them are studied in Section 4. Some relevant remarks close the paper. 


\section{Classical Poincaré algebra in a null-plane basis}

Hereafter we shall consider the null-plane "orthogonal" to the light-like vector $n=$ $\left(\frac{1}{2}, 0,0, \frac{1}{2}\right)$ as our initial surface [2]. A coordinate system well adapted to null-planes $\Pi_{n}^{\tau}$ is given by

$$
x^{-}=n \cdot x=\frac{1}{2}\left(x^{0}-x^{3}\right)=\tau, \quad x^{+}=x^{0}+x^{3}, \quad x_{T}=\left(x^{1}, x^{2}\right) .
$$

Hence, a point $x$ contained in the null-plane $\Pi_{n}^{\tau}$ will be labelled by the coordinates $\left(x^{+}, x^{1}, x^{2}\right)$. The remaining coordinate $x^{-}$plays the role of a time. A particular nullplane is $\Pi_{n}^{0},(\tau=0)$, i.e., $x^{-}=n \cdot x=0$; this plane is mapped onto itself by the boosts generated by $K_{3}$, which leave the transverse coordinates $x_{T}=\left(x^{1}, x^{2}\right)$ unchanged and $e^{\chi K_{3}}$ maps $x_{+}$on $e^{\chi} x_{+}$. The Poincaré algebra generators can be classified according to their transformation property with respect to $K_{3}$; any generator $A$ obeying

$$
\left[K_{3}, A\right]=\gamma A,
$$

is referred to as an operator of "goodness" $\gamma$. The ten generators of $\mathcal{P}(3+1)$ (adapted to the null-plane coordinates in terms of the usual physical basis) can be listed in relation to their goodness in the following way:

$$
\begin{array}{cccc}
\gamma=+1: & E_{1}=\frac{1}{2}\left(K_{1}+J_{2}\right), & E_{2}=\frac{1}{2}\left(K_{2}-J_{1}\right), & P_{+}=\frac{1}{2}\left(P_{0}+P_{3}\right) ; \\
\gamma=0: & K_{3}, \quad J_{3}, & P_{1}, \quad P_{2} ; \\
\gamma=-1: & F_{1}=\frac{1}{2}\left(K_{1}-J_{2}\right), & F_{2}=\frac{1}{2}\left(K_{2}+J_{1}\right), & P_{-}=\frac{1}{2}\left(P_{0}-P_{3}\right) .
\end{array}
$$

The non-vanishing Lie brackets of $\mathcal{P}(3+1)$ are $(i, j=1,2)$ :

$$
\begin{aligned}
& {\left[K_{3}, P_{+}\right]=P_{+}, \quad\left[K_{3}, P_{-}\right]=-P_{-}, \quad\left[K_{3}, E_{i}\right]=E_{i}, \quad\left[K_{3}, F_{i}\right]=-F_{i},} \\
& {\left[J_{3}, P_{i}\right]=-\varepsilon_{i j 3} P_{j}, \quad\left[J_{3}, E_{i}\right]=-\varepsilon_{i j 3} E_{j}, \quad\left[J_{3}, F_{i}\right]=-\varepsilon_{i j 3} F_{j},} \\
& {\left[E_{i}, P_{j}\right]=\delta_{i j} P_{+}, \quad\left[F_{i}, P_{j}\right]=\delta_{i j} P_{-}, \quad\left[P_{+}, F_{i}\right]=-P_{i},} \\
& {\left[E_{i}, F_{j}\right]=\delta_{i j} K_{3}+\varepsilon_{i j 3} J_{3}, \quad\left[P_{-}, E_{i}\right]=-P_{i} .}
\end{aligned}
$$

The set of all Poincaré generators with the same goodness $\gamma$ spans a subgroup $G_{\gamma}$ (in particular, the subgroups $G_{ \pm 1}$ are Abelian). Moreover, there exist two sevenparametrical Poincaré subgrups with a semidirect product structure: $S_{ \pm}=G_{0} \odot G_{ \pm 1}$. The stability group of the plane $\Pi_{n}^{0}$ coincides with the group $S_{+}$. The remaining three generators, which originate $G_{-1}$, act on $\Pi_{n}^{0}$ as follows: the generator $P_{-}$translates $\Pi_{n}^{0}$ into $\Pi_{n}^{\tau}$, while the generators $F_{i}$ rotate it around the surface of the light-cone $x^{2}=0$. Therefore, if $x^{-}=\tau$ is considered as an evolution parameter, then $P_{-}$and

$F_{i}$ describe the dynamical evolution from the null-plane $x^{-}=0$; they are called Hamiltonians.

The two Casimirs of $\mathcal{P}(3+1)$ are given by:

$$
\begin{aligned}
& M^{2}=2 P_{-} P_{+}-P_{1}^{2}-P_{2}^{2}, \\
& W^{2}=W_{13}^{2}+W_{23}^{2}+W_{+} W_{-}+W_{-} W_{+},
\end{aligned}
$$


where

$$
\begin{aligned}
& W_{13}=K_{3} P_{1}+E_{1} P_{-}-F_{1} P_{+}, \quad W_{23}=K_{3} P_{2}+E_{2} P_{-}-F_{2} P_{+}, \\
& W_{+}=E_{1} P_{2}-E_{2} P_{1}+J_{3} P_{+}, \quad W_{-}=F_{1} P_{2}-F_{2} P_{1}+J_{3} P_{-} .
\end{aligned}
$$

The second-order Casimir $M^{2}$ is the square of the mass operator $(M)$ and the fourthorder one $W^{2}$ is the square of the Pauli-Lubanski operator. Lie brackets involving the components $W_{i}$ and the null-plane generators of $\mathcal{P}(3+1)$ are given in Appendix A.

\section{Quantum Poincaré algebra in a null-plane basis}

In [9], the standard deformation of $\mathcal{P}(3+1)$ has been derived by using the socalled deformation embedding method, a kind of quantum analogue of the classical embedding of the $\mathcal{P}(2+1)$ algebra into $\mathcal{P}(3+1)$ (see also Ref. [11] for arbitrary dimension). In this way, the $(2+1)$ dimensional deformation of a given (affine) algebra turns out to be the cornerstone for the obtention of its higher dimensional generalizations. Since a null-plane quantum $\mathcal{P}(2+1)$ algebra has been given in [10], the same procedure can be now applied as follows:

1) Let us look for classical subalgebras of $\mathcal{P}(3+1)$ isomorphic to the null-plane algebra $\mathcal{P}(2+1)$ : there exist two of them. Namely,

$$
\Pi_{13}=\left\langle K_{3}, E_{1}, F_{1}, P_{1}, P_{+}, P_{-}\right\rangle, \quad \Pi_{23}=\left\langle K_{3}, E_{2}, F_{2}, P_{2}, P_{+}, P_{-}\right\rangle .
$$

2) We assume that $U_{z} \Pi_{13}$ and $U_{z} \Pi_{23}$, endowed with the null-plane $(2+1)$ deformation of [10] are "restrictions" [11] of the quantization for $U_{z} \mathcal{P}(3+1)$.

3) Consider the simplest Ansatz for the coproduct and commutation rules of $U_{z} \mathcal{P}(3+1)$ consistent with these restrictions.

4) Check the selfconsistency of the structure obtained in 3) by imposing that the coproduct is a Hopf algebra homomorphism.

The final result is the following null-plane deformation of $U_{z} \mathcal{P}(3+1)$, with coproduct given by:

$$
\begin{aligned}
\Delta(X)= & 1 \otimes X+X \otimes 1, \quad \text { for } \quad X \in\left\{P_{+}, E_{1}, E_{2}, J_{3}\right\} \\
\Delta(Y)= & e^{-z P_{+}} \otimes Y+Y \otimes e^{z P_{+}}, \quad \text { for } \quad Y \in\left\{P_{-}, P_{1}, P_{2}\right\}, \\
\Delta\left(F_{1}\right)= & e^{-z P_{+}} \otimes F_{1}+F_{1} \otimes e^{z P_{+}}+z e^{-z P_{+}} E_{1} \otimes P_{-}-z P_{-} \otimes E_{1} e^{z P_{+}} \\
& +z e^{-z P_{+}} J_{3} \otimes P_{2}-z P_{2} \otimes J_{3} e^{z P_{+}} \\
\Delta\left(F_{2}\right)= & e^{-z P_{+}} \otimes F_{2}+F_{2} \otimes e^{z P_{+}}+z e^{-z P_{+}} E_{2} \otimes P_{-}-z P_{-} \otimes E_{2} e^{z P_{+}} \\
& +z e^{-z P_{+}} J_{3} \otimes P_{1}-z P_{1} \otimes J_{3} e^{z P_{+}} \\
\Delta\left(K_{3}\right)= & e^{-z P_{+}} \otimes K_{3}+K_{3} \otimes e^{z P_{+}}+z e^{-z P_{+}} E_{1} \otimes P_{1}-z P_{1} \otimes E_{1} e^{z P_{+}} \\
& +z e^{-z P_{+}} E_{2} \otimes P_{2}-z P_{2} \otimes E_{2} e^{z P_{+}} ;
\end{aligned}
$$

commutation relations:

$$
\left[K_{3}, P_{+}\right]=\frac{\sinh z P_{+}}{z}, \quad\left[K_{3}, P_{-}\right]=-P_{-} \cosh z P_{+}, \quad\left[K_{3}, E_{i}\right]=E_{i} \cosh z P_{+},
$$




$$
\begin{aligned}
& {\left[K_{3}, F_{1}\right]=-F_{1} \cosh z P_{+}+z E_{1} P_{-} \sinh z P_{+}-z^{2} P_{2} W_{+}^{q},} \\
& {\left[K_{3}, F_{2}\right]=-F_{2} \cosh z P_{+}+z E_{2} P_{-} \sinh z P_{+}+z^{2} P_{1} W_{+}^{q},} \\
& {\left[J_{3}, P_{i}\right]=-\varepsilon_{i j 3} P_{j}, \quad\left[J_{3}, E_{i}\right]=-\varepsilon_{i j 3} E_{j}, \quad\left[J_{3}, F_{i}\right]=-\varepsilon_{i j 3} F_{j},} \\
& {\left[E_{i}, P_{j}\right]=\delta_{i j} \frac{\sinh z P_{+}}{z}, \quad\left[F_{i}, P_{j}\right]=\delta_{i j} P_{-} \cosh z P_{+},} \\
& {\left[E_{i}, F_{j}\right]=\delta_{i j} K_{3}+\varepsilon_{i j 3} J_{3} \cosh z P_{+}, \quad\left[P_{+}, F_{i}\right]=-P_{i},} \\
& {\left[F_{1}, F_{2}\right]=z^{2} P_{-} W_{+}^{q}+z P_{-} J_{3} \sinh z P_{+}, \quad\left[P_{-}, E_{i}\right]=-P_{i} .}
\end{aligned}
$$

(the quantum component $W_{+}^{q}$ of the Pauli-Lubanski vector is defined below (3. 9)); counit and antipode:

$$
\epsilon(X)=0 ; \quad \gamma(X)=-e^{3 z P_{+}} X e^{-3 z P_{+}}, \quad \text { for } X \in\left\{P_{ \pm}, P_{i}, E_{i}, F_{i}, J_{3}\right\} .
$$

As a byproduct of the embedding method, the underlying coboundary Lie bialgebra is also obtained. Explicitly, the classical $r$-matrix

$$
r=2 z\left(K_{3} \wedge P_{+}+E_{1} \wedge P_{1}+E_{2} \wedge P_{2}\right),
$$

is a skew-solution of the CYBE (it can be identified with one appearing in Zakrzewski's classification [12]), and generates the cocommutator $\delta(X)=[1 \otimes X+$ $X \otimes 1, r]$ that provides the first order term in $z$ within the coproduct $(3.2)$ :

$$
\begin{aligned}
& \delta(X)=0, \quad \text { for } \quad X \in\left\{P_{+}, E_{1}, E_{2}, J_{3}\right\}, \\
& \delta(Y)=2 z Y \wedge P_{+}, \quad \text { for } Y \in\left\{P_{-}, P_{1}, P_{2}\right\}, \\
& \delta\left(F_{1}\right)=2 z\left(F_{1} \wedge P_{+}+E_{1} \wedge P_{-}+J_{3} \wedge P_{2}\right), \\
& \delta\left(F_{2}\right)=2 z\left(F_{2} \wedge P_{+}+E_{2} \wedge P_{-}+J_{3} \wedge P_{1}\right), \\
& \delta\left(K_{3}\right)=2 z\left(K_{3} \wedge P_{+}+E_{1} \wedge P_{1}+E_{2} \wedge P_{2}\right) .
\end{aligned}
$$

The mass (2. 5) and the Pauli-Lubanski (2. 6) operators can be deformed as follows:

$$
\begin{gathered}
M_{q}^{2}=2 P_{-} \frac{\sinh z P_{+}}{z}-P_{1}^{2}-P_{2}^{2}, \\
W_{q}^{2}=\left(W_{13}^{q}\right)^{2}+\left(W_{23}^{q}\right)^{2}+\cosh z P_{+}\left\{W_{+}^{q} W_{-}^{q}+W_{-}^{q} W_{+}^{q}\right\}-z^{2} M_{q}^{2}\left(W_{+}^{q}\right)^{2},
\end{gathered}
$$

where

$$
\begin{aligned}
& W_{13}^{q}=K_{3} P_{1}+E_{1} P_{-} \cosh z P_{+}-F_{1} \frac{\sinh z P_{+}}{z}, \\
& W_{23}^{q}=K_{3} P_{2}+E_{2} P_{-} \cosh z P_{+}-F_{2} \frac{\sinh ^{2} P_{+}}{z}, \\
& W_{+}^{q}=E_{1} P_{2}-E_{2} P_{1}+J_{3} \frac{\sinh z P_{+}}{z}, \\
& W_{-}^{q}=F_{1} P_{2}-F_{2} P_{1}+J_{3} P_{-} \cosh z P_{+} .
\end{aligned}
$$

The commutation rules between the components $W_{i}^{q}$ and the Hopf algebra generators are given in Appendix A; they show that $W_{q}^{2}$ belongs to the center of $U_{z} \mathcal{P}(3+1)$ as $M_{q}^{2}$ 
It is interesting to analyse now the deformed algebras given by the generators of the classical subgroups $G_{\gamma}, \gamma=-1,0,+1$ and $S_{ \pm}$. It is a rather remarkable fact that the generators of the null-plane stability group, $S_{+}$, determine a deformed Hopf subalgebra of $U_{z} \mathcal{P}(3+1)$ (that we shall denote by $U_{z} S_{+}$), while the Lie subalgebra generating $G_{+1}$ gives a trivial (i.e., non deformed) Hopf subalgebra under deformation. On the other hand, $G_{0}, G_{-1}$ and $S_{-}$do not originate Hopf subalgebras. Moreover, although the classical group $G_{-1}$ is Abelian, this is not the case in the quantum version.

There is another physically interesting deformed Hopf subalgebra in $U_{z} \mathcal{P}(3+1)$ : the set of generators $\left\langle J_{3}, E_{1}, E_{2}, P_{1}, P_{2}, P_{+}, P_{-}\right\rangle$generates a quantum deformation, $U_{z} \widetilde{\mathcal{G}}(2+1)$, of the $(2+1)$ extended Galilean algebra. The generator $P_{+}$is central, and the structure of $U_{z} \widetilde{\mathcal{G}}(2+1)$ can be studied by considering a new physical basis $\left\{\tilde{H}, \tilde{P}_{1}, \tilde{P}_{2}, \tilde{K}_{1}, \tilde{K}_{2}, \tilde{J}\right\}$ linked, as usual, to the time translation, space translations, boosts and space rotation, respectively. For the Galilean case we introduce a central element $\tilde{M}$ which can be identified with the mass of a free particle. Explicitly, the relations between the null-plane and the usual kinematical bases for this subalgebra are:

$U_{z} \widetilde{\mathcal{G}}(2+1):\left\langle J_{3}, E_{1}, E_{2}, P_{1}, P_{2}, P_{-}, P_{+}\right\rangle \equiv\left\langle-\tilde{J}_{3}, \tilde{K}_{1}, \tilde{K}_{2}, \tilde{P}_{1}, \tilde{P}_{2}, \tilde{H}, \tilde{M}\right\rangle$.

The Hopf structure of $U_{z} \widetilde{\mathcal{G}}(2+1)$ has the following coproduct and non-vanishing commutation rules:

$$
\begin{gathered}
\Delta(X)=1 \otimes X+X \otimes 1, \quad X \in\left\{\tilde{M}, \tilde{J}_{3}, \tilde{K}_{1}, \tilde{K}_{2}\right\} \\
\Delta(Y)=e^{-z \tilde{M}} \otimes Y+Y \otimes e^{z \tilde{M}}, \quad Y \in\left\{\tilde{H}, \tilde{P}_{1}, \tilde{P}_{2}\right\} \\
{\left[\tilde{J}_{3}, \tilde{K}_{i}\right]=\varepsilon_{i j 3} \tilde{K}_{j}, \quad\left[\tilde{J}_{3}, \tilde{P}_{i}\right]=\varepsilon_{i j 3} \tilde{P}_{j},} \\
{\left[\tilde{K}_{i}, \tilde{H}\right]=\tilde{P}_{i}, \quad\left[\tilde{K}_{i}, \tilde{P}_{j}\right]=\delta_{i j} \frac{\sinh z \tilde{M}}{z} .}
\end{gathered}
$$

The center of $U_{z} \widetilde{\mathcal{G}}(2+1)$ is generated by the mass $\tilde{M}$, the energy $E_{q}$ and the intrinsic angular momentum $L_{q}$ :

$$
\begin{aligned}
& E_{q}^{2}=\tilde{P}_{1}^{2}+\tilde{P}_{2}^{2}-\tilde{H} \frac{\sinh z \tilde{M}}{z} \\
& L_{q}=\tilde{K}_{1} \tilde{P}_{2}-\tilde{K}_{2} \tilde{P}_{1}-\tilde{J}_{3} \frac{\sinh z \tilde{M}}{z} .
\end{aligned}
$$

\section{Spin, Hamiltonians and Position Operators}

In the classical Poincaré algebra the spin $\overrightarrow{\mathcal{S}}$ satisfies the commutation rules:

$$
\left[\mathcal{S}_{i}, \mathcal{S}_{j}\right]=\varepsilon_{i j k} \mathcal{S}_{k}, \quad\left[M, \mathcal{S}_{i}\right]=0, \quad i, j, k=1,2,3,
$$


where the components $\mathcal{S}_{i}$ are given in terms of the Pauli-Lubanski vector by

$$
M \mathcal{S}_{1}=W_{23}+\left(P_{1} / P_{+}\right) W_{+}, \quad M \mathcal{S}_{2}=W_{13}-\left(P_{2} / P_{+}\right) W_{+}, \quad \mathcal{S}_{3}=W_{+} / P_{+}
$$

The spin $\overrightarrow{\mathcal{S}}$ commutes with all the generators of the stability group $S_{+}$except $J_{3}$. In particular, the component $\mathcal{S}_{3}$ is a central element of $S_{+}$. Its eigenvalue $h$ is called the null-plane helicity.

Relations (4. 2) enable us to write the Hamiltonians $P_{-}, F_{1}$ and $F_{2}$ in terms of the mass and spin operators:

$$
\begin{aligned}
& P_{-}=\left[M^{2}+P_{1}^{2}+P_{2}^{2}\right]\left(1 / 2 P_{+}\right), \\
& F_{1}=\left[K_{3} P_{1}+E_{1} P_{-}-\left(M \mathcal{S}_{2}+P_{2} \mathcal{S}_{3}\right)\right]\left(1 / P_{+}\right), \\
& F_{2}=\left[K_{3} P_{2}+E_{2} P_{-}-\left(M \mathcal{S}_{1}-P_{1} \mathcal{S}_{3}\right)\right]\left(1 / P_{+}\right) .
\end{aligned}
$$

In these expressions the most relevant objects (rather than $\mathcal{S}_{1}, \mathcal{S}_{2}$ and $M$ ) are the products $M \mathcal{S}_{1}, M \mathcal{S}_{2}$ and $M^{2}$. These quadratic elements are called "reduced Hamiltonians".

\subsection{Quantum Spin and Hamiltonians}

The quantum counterpart of this classical structure can be straightforwardly obtained once we know the $q$-Casimirs of $U_{z} \mathcal{P}(3+1)\left(\begin{array}{l}3.7 \\ 3.9\end{array}\right)$. We define the components $\mathcal{S}_{i}^{q}$ of the quantum spin in such a way that the following relations hold:

$$
M_{q} \mathcal{S}_{1}^{q}=W_{23}^{q}+\frac{z P_{1}}{\tanh z P_{+}} W_{+}^{q}, \quad M_{q} \mathcal{S}_{2}^{q}=W_{13}^{q}-\frac{z P_{2}}{\tanh z P_{+}} W_{+}^{q}, \quad \mathcal{S}_{3}^{q}=\frac{z W_{+}^{q}}{\sinh z P_{+}} .
$$

Relations A.3 A.7) of Appendix A show that the $q$-spin $\overrightarrow{\mathcal{S}^{q}}$, so introduced, verifies the following commutation rules:

$$
\left[\mathcal{S}_{i}^{q}, \mathcal{S}_{j}^{q}\right]=\varepsilon_{i j k} \mathcal{S}_{k}^{q}, \quad\left[M_{q}, \mathcal{S}_{i}^{q}\right]=0
$$

Similarly to the classical case, $\overrightarrow{\mathcal{S}}^{q}$ commutes with all the generators of the quantum stability group $U_{z} S_{+}$except $J_{3}$; furthermore, the component $\mathcal{S}_{3}^{q}$ (quantum helicity) is a central element for $U_{z} S_{+}$.

The Hamiltonians now adopt the following deformed expressions:

$$
\begin{aligned}
& P_{-}=\left[M_{q}^{2}+P_{1}^{2}+P_{2}^{2}\right] \frac{z}{2 \sinh z P_{+}} \\
& F_{1}=\left[K_{3} P_{1}+E_{1} P_{-} \cosh z P_{+}-\left(M_{q} \mathcal{S}_{2}^{q}+P_{2} \cosh z P_{+} \mathcal{S}_{3}^{q}\right)\right] \frac{z}{\sinh z P_{+}} \\
& F_{2}=\left[K_{3} P_{2}+E_{2} P_{-} \cosh z P_{+}-\left(M_{q} \mathcal{S}_{1}^{q}-P_{1} \cosh z P_{+} \mathcal{S}_{3}^{q}\right)\right] \frac{z}{\sinh z P_{+}}
\end{aligned}
$$

and we call the products $M_{q} \mathcal{S}_{1}^{q}, M_{q} \mathcal{S}_{2}^{q}$ and $M_{q}^{2}$ quantum reduced Hamiltonians. Note that in the quantum case $\left[F_{1}, F_{2}\right] \neq 0$. 
A quantum differential realization for $U_{z} \mathcal{P}(3+1)$ can be obtained in the momentum representation with coordinates $\mathbf{p}=\left(p_{+}, p_{1}, p_{2}\right)=\left(p_{+}, p_{T}\right)$ and $p_{+}>0$. The simplest procedure to derive it consists in getting firstly a realization for the quantum stability group $U_{z} S_{+}$. It is easy to check that it reads

$$
\begin{array}{ll}
P_{+}=p_{+}, \quad P_{i}=p_{i}, & E_{i}=\frac{\sinh z p_{+}}{z} \partial_{i}, \\
J_{3}=p_{1} \partial_{2}-p_{2} \partial_{1}+\mathcal{S}_{3}^{q}, & K_{3}=\frac{\sinh z p_{+}}{z} \partial_{+}, \quad i=1,2,
\end{array}
$$

where $\partial_{i}=\frac{\partial}{\partial p_{i}}$. By introducing (4.7) in the expressions of the quantum Hamiltonians (4.6) we get

$$
\begin{aligned}
& P_{-}=\frac{z\left(M_{q}^{2}+p_{T}^{2}\right)}{2 \sinh z p_{+}}, \\
& F_{1}=p_{1} \partial_{+}+\frac{z\left(M_{q}^{2}+p_{T}^{2}\right)}{2 \tanh z p_{+}} \partial_{1}-\frac{z}{\sinh z p_{+}}\left(M_{q} \mathcal{S}_{2}^{q}+p_{2} \cosh z p_{+} \mathcal{S}_{3}^{q}\right), \\
& F_{2}=p_{2} \partial_{+}+\frac{z\left(M_{q}^{2}+p_{T}^{2}\right)}{2 \tanh z p_{+}} \partial_{2}-\frac{z}{\sinh z p_{+}}\left(M_{q} \mathcal{S}_{1}^{q}-p_{1} \cosh z p_{+} \mathcal{S}_{3}^{q}\right),
\end{aligned}
$$

with $p_{T}^{2}=p_{1}^{2}+p_{2}^{2}$.

Let us consider a basis vectors $|\mathbf{p}, h\rangle$ for this representation, i.e., $P_{l}|\mathbf{p}, h\rangle=$ $p_{l}|\mathbf{p}, h\rangle, l=+, 1,2$, and $S_{3}|\mathbf{p}, h\rangle=h|\mathbf{p}, h\rangle$. The action of the remaining operators is given by (4.7) and (4.8). The norm of these eigenvectors is

$$
\left\langle p^{\prime}, h \mid p, h\right\rangle=(2 \pi)^{3} 2 \frac{\sinh z p_{+}}{z} \delta\left(p^{\prime}-p\right) \delta_{h^{\prime}, h} .
$$

Now, we introduce a quantum inner product of two arbitrary states depending on the three momentum coordinates $\mathbf{p}=\left(p_{+}, p_{1}, p_{2}\right)$ and the helicity $h$ (the quantum spin has classical commutation rules):

$$
\langle\phi \mid \psi\rangle=\frac{1}{(2 \pi)^{3}} \sum_{h} \int \frac{z d^{3} \mathbf{p}}{2 \sinh z p_{+}} \phi_{h}^{*}(\mathbf{p}) \psi_{h}(\mathbf{p}),
$$

where $\phi_{h}(\mathbf{p})=<p, h \mid \phi>$. Note that in the limit $z \rightarrow 0$ we recover the appropiate classical inner product. According to expression (4. 10), generators $P_{+}$, and $P_{l}$ are hermitian operators while $K_{3}, J_{3}$ and $E_{l}$ are skew-hermitian. Therefore,

$$
\hat{P}_{+} \equiv P_{+}, \quad \hat{P}_{l} \equiv P_{l}, \quad \hat{K}_{3} \equiv i K_{3}, \quad \hat{E}_{l} \equiv i E_{l}, \quad \hat{J}_{3} \equiv i J_{3}, \quad(l=1,2),
$$

gives a hermitian representation of $U_{z} S_{+}$relative to (4.10) which induces a hermitian representation of whole $q$-algebra $U_{z}(\mathcal{P}(3+1)$. Note that the commutations rules for the hermitian operators (4.11) are also given by (3.3) by adding the imaginary unit $i$ in the r.h.s of the corresponding commutators. Only $P_{-}$out of the remaining three generators is hermitian. On the other hand, let us also remark that in order to extend the $*$-operation at the Hopf algebra level $z$ has to be consider a dimensional real parameter $[z]=\left[p_{+}\right]^{-1}$ (see coproduct $\left.(3.2)\right)$. 
We can consider the coordinate $x^{-}$as a "time" $(\tau)$, and the complete wave function will be $\tau$-dependent $f_{h}(\mathbf{p}, \tau)$. The Hamiltonian $\hat{P}_{-} \equiv P_{-}$provides the evolution of the null-plane in terms of a $q$-deformed Schrödinger equation $i \partial_{\tau} f_{h}(\mathbf{p}, \tau)=$ $\hat{P}_{-} f_{h}(\mathbf{p}, \tau)$. From the representation $(4.8)$ we obtain

$$
i \partial_{\tau} \psi_{h}(\mathbf{p}, \tau)=\frac{z\left(m_{q}^{2}+p_{T}^{2}\right)}{2 \sinh z p_{+}} \psi_{h}(\mathbf{p}, \tau),
$$

where the functions $\psi_{h}(\mathbf{p}, \tau)$ are square integrable under (4. 10)

$$
\frac{1}{(2 \pi)^{3}} \sum_{h} \int \frac{z d^{3} \mathbf{p}}{2 \sinh z p_{+}}\left|\psi_{h}(\mathbf{p}, \tau)\right|^{2}<+\infty .
$$

The r.h.s. of this $q$-Schrödinger equation can be seen as a deformation of the kinetic term $H_{0}=\left(m_{q}^{2}+p_{T}^{2}\right) / 2 p_{+}$of the null-plane bound state equation in quantum chromodynamics [4, 6]. In fact, when $z$ is small enough, we can write

$$
\hat{P}_{-}=\frac{z\left(m_{q}^{2}+p_{T}^{2}\right)}{2 \sinh z p_{+}}=\frac{m_{q}^{2}+p_{T}^{2}}{2 p_{+}}-\frac{1}{12} z^{2} p_{+}\left(m_{q}^{2}+p_{T}^{2}\right)+o\left(z^{4}\right)=H_{0}+V_{z},
$$

and the null-plane deformation intrinsically includes a dynamical part $V_{z}$ to be computed together with the quantum chromodynamics interaction.

\subsection{Quantum Position Operators}

In the classical case there exist two null-plane (or mean transverse) position operators $\hat{Q}_{i}(i=1,2)$ (see [2]) which measure the position of the center of the longitudinal momentum. Explicitly,

$$
\hat{Q}_{i}=\hat{E}_{i} / \hat{P}_{+}, \quad i=1,2
$$

and they satisfy the following classical commutation rules with the generators of the stability group $S_{+}$:

$$
\begin{array}{lc}
{\left[\hat{Q}_{i}, \hat{E}_{j}\right]=0,} & {\left[\hat{Q}_{i}, \hat{K}_{3}\right]=0, \quad\left[\hat{Q}_{i}, \hat{J}_{3}\right]=i \varepsilon_{i j 3} \hat{Q}_{j}} \\
{\left[\hat{Q}_{i}, \hat{P}_{+}\right]=0,} & {\left[\hat{Q}_{i}, \hat{P}_{j}\right]=i \delta_{i j}, \quad\left[\hat{Q}_{1}, \hat{Q}_{2}\right]=0 .}
\end{array}
$$

From the definition of $\hat{Q}_{i}$ (4. 15) in the classical version of the representation (4. 7) the position operators are $\hat{Q}_{i}=i \frac{\partial}{\partial p_{i}}$. They commute with the reduced Hamiltonians $M^{2}, M \mathcal{S}_{i}$ and with the helicity $\mathcal{S}_{3}$. The transverse velocity operator $\left(\frac{d}{d \tau} \hat{Q}_{i}\right)$ is given by the commutator of $\hat{Q}_{i}$ with the Hamiltonian $\hat{P}_{-}$:

$$
\frac{d}{d \tau} \hat{Q}_{i}=\left[\hat{Q}_{i}, \hat{P}_{-}\right]=i \hat{P}_{i} / \hat{P}_{+}
$$

In order to find quantum deformations for the position operators, $\hat{Q}_{i}$, we make the following Ansatz

$$
\hat{Q}_{i}=\hat{E}_{i} / f\left(z, \hat{P}_{+}\right), \quad i=1,2,
$$


where $f\left(z, \hat{P}_{+}\right)$is an arbitrary (smooth) function such that $\lim _{z \rightarrow 0} f\left(z, \hat{P}_{+}\right)=\hat{P}_{+}$ and $f\left(z, p_{+}\right) \neq 0$ for $z$ real and $p_{+}>0$. Commutators (4. 16) and (4. 17) become in the quantum case (we omit the arguments in the function $f$ ):

$$
\begin{aligned}
& {\left[\hat{Q}_{i}, \hat{E}_{j}\right]=0, \quad\left[\hat{Q}_{i}, \hat{K}_{3}\right]=i \hat{E}_{i}\left\{\frac{f^{\prime}}{f^{2}} \frac{\sinh z \hat{P}_{+}}{z}-\frac{\cosh z \hat{P}_{+}}{f}\right\}, \quad\left[\hat{Q}_{i}, \hat{J}_{3}\right]=i \varepsilon_{i j 3} \hat{Q}_{j},} \\
& {\left[\hat{Q}_{i}, \hat{P}_{+}\right]=0, \quad\left[\hat{Q}_{i}, \hat{P}_{j}\right]=i \delta_{i j} \frac{\sinh z \hat{P}_{+}}{z} \frac{1}{f}, \quad\left[\hat{Q}_{1}, \hat{Q}_{2}\right]=0, \quad\left[\hat{Q}_{i}, \hat{P}_{-}\right]=i \frac{\hat{P}_{i}}{f}, \quad}
\end{aligned}
$$

where $f^{\prime}$ is "the formal derivative" of $f$ with respect to $\hat{P}_{+}$. Moreover, whatever the choice of the function $f$ be, the operators $\hat{Q}_{i}$ are hermitian with respect to (4. 10), and they commute with the quantum reduced Hamiltonians and with the quantum helicity -similarly to the classical case. We analyze the most natural possibilities:

(1) $f=\sinh z \hat{P}_{+} / z$. The only deformed bracket in (4. 19) is the one corresponding to the transverse velocity operator, i.e., $\left[\hat{Q}_{i}, \hat{P}_{-}\right]=i z \hat{P}_{i} / \sinh z \hat{P}_{+}$. Thus, the quantum realization for $\hat{Q}_{i}$ is

$$
\hat{Q}_{i}=\frac{z \hat{E}_{i}}{\sinh z \hat{P}_{+}},
$$

which, in terms of the differential realization (4.7) provides the undeformed result $\hat{Q}_{i} \equiv i \frac{\partial}{\partial p_{i}}$ for the position operators. As a result, the "generalized" uncertainty principle derived from (4.20) is given by

$$
\Delta \hat{Q}_{i} \Delta \hat{P}_{i} \geq \frac{1}{2}
$$

(in this case $\left[\hat{Q}_{i}, \hat{P}_{j}\right]=i \delta_{i j}$, since we have taken $\hbar=c=1$ ).

(2) $f=\tanh z \hat{P}_{+} / z$. Now the commutation rules (4. 19) read:

$$
\begin{gathered}
{\left[\hat{Q}_{i}, \hat{E}_{j}\right]=0, \quad\left[\hat{Q}_{i}, \hat{K}_{3}\right]=i \hat{E}_{i} z \sinh z \hat{P}_{+}, \quad\left[\hat{Q}_{i}, \hat{J}_{3}\right]=i \varepsilon_{i j 3} \hat{Q}_{j}} \\
{\left[\hat{Q}_{i}, \hat{P}_{+}\right]=0, \quad\left[\hat{Q}_{i}, \hat{P}_{j}\right]=i \delta_{i j} \cosh z \hat{P}_{+}, \quad\left[\hat{Q}_{1}, \hat{Q}_{2}\right]=0 .}
\end{gathered}
$$

The time derivative of $\hat{Q}_{i}$ turns into

$$
\frac{d}{d \tau} \hat{Q}_{i}=\left[\hat{Q}_{i}, \hat{P}_{-}\right]=i \frac{z \hat{P}_{i}}{\tanh z \hat{P}_{+}},
$$

and the corresponding generalized uncertainty relation is given by

$$
\Delta \hat{Q}_{i} \Delta \hat{P}_{i} \geq \frac{1}{2} \Delta\left(\cosh z \hat{P}_{+}\right) .
$$

A power series expansion gives

$$
\Delta \hat{Q}_{i} \Delta \hat{P}_{i} \geq \frac{1}{2}\left(1+\frac{z^{2}}{2}\left(\Delta \hat{P}_{+}\right)^{2}+\ldots\right),
$$

a result similar to the one presented in [13] that concerns a generalized uncertainty principle in Quantum Gravity. In our case the "classical" value $\Delta \hat{Q}_{i} \Delta \hat{P}_{i} \geq \frac{1}{2}$ appears deformed by addition of terms depending on $\Delta \hat{P}_{+}$. 


\section{Concluding remarks}

We have presented a new quantum deformation of the algebra $\mathcal{P}(3+1)$ whose natural framework is the null-plane basis for this algebra. From the point of view of quantum group theory, this deformation presents some interesting properties that distinguish it from the other ones already known. The essential point is that the classical $r$-matrix (3. 5) is triangular; thus, a $*_{\hbar}$-product quantizing the algebra of representative functions on the group exists. First order information concerning the corresponding null-plane quantum Poincaré group can be extracted from the Lie bialgebra structure (3.6). In particular, for the (now non-commutative) coordinates $\left(\hat{p}_{1}, \hat{p}_{2}, \hat{p}_{+}\right)$we shall have

$$
\left[\hat{p}_{1}, \hat{p}_{+}\right]=2 z \hat{p}_{1}+o\left(z^{2}\right), \quad\left[\hat{p}_{2}, \hat{p}_{+}\right]=2 z \hat{p}_{2}+o\left(z^{2}\right)
$$

Moreover, since $r$ is a solution of the CYBE, a universal $R$-matrix turning this nonstandard deformation into a triangular Hopf algebra could be hopefully derived. All these formal aspects deserve further study.

From a physical point of view, the most relevant feature of the results here displayed is the parallelism between the classical subalgebras and the Hopf algebra ones induced after deformation. This fact is the cornerstone behind the straightforward generalization of the classical constructions intrinsically containing a modification of both the kinematical and dynamical classical symmetries. In this way, by means of a hermitian representation of the quantum isotopy algebra of the null-plane $\Pi_{n}^{0}$, it has been possible to define a family of (hermitian) Newton-Wigner position operators corresponding to the transverse coordinates $q_{1}$ and $q_{2}$. These operators are not univocally defined as there exists some freedom in the choice of a function $f\left(z, \hat{P}_{+}\right)$ given in the above section. The Lie bracket between the position and the momentum operators gives rise to a $q$-deformed generalized uncertainty principle whose expression is

$$
\Delta \hat{Q}_{i} \Delta \hat{P}_{i} \geq \frac{1}{2} \Delta\left(\frac{\sinh z \hat{P}_{+}}{z f\left(z, \hat{P}_{+}\right)}\right) .
$$

Note that expressions (4.21) and (4.24) are particular cases of (5.2) according to the different choices of $f$.

The time evolution of a null-plane deformed system (given by the $q$-Schrödinger equation (4.12)) is also a consecuence of the existence of an extended $(2+1)$ Galilean Hopf subalgebra, in which $\hat{P}_{-}$plays the role of the deformed Hamiltonian and $\hat{P}_{+}$ is the mass operator in perfect agreement with the classical version. Quantum deformed reformulations of classical models based in the null-plane description (and in particular, the role of the deformed coproduct for composite systems) seem to be worth studying. 


\section{Appendix A: Commutation relations for the classical and quantum Pauli-Lubanski operator}

The non-vanishing commutation relations of the components $W_{i}$ of the PauliLubanski vector (2.7) among themselves and with the generators of $\mathcal{P}(3+1)$ are:

$$
\begin{aligned}
& {\left[J_{3}, W_{13}\right]=-W_{23}, \quad\left[E_{2}, W_{13}\right]=W_{+}, \quad\left[F_{2}, W_{13}\right]=-W_{-},} \\
& {\left[J_{3}, W_{23}\right]=W_{13}, \quad\left[E_{1}, W_{23}\right]=-W_{+}, \quad\left[F_{1}, W_{23}\right]=W_{-},} \\
& {\left[E_{1}, W_{-}\right]=W_{23}, \quad\left[E_{2}, W_{-}\right]=-W_{13}, \quad\left[K_{3}, W_{-}\right]=-W_{-},} \\
& {\left[F_{1}, W_{+}\right]=-W_{23}, \quad\left[F_{2}, W_{+}\right]=W_{13}, \quad\left[K_{3}, W_{+}\right]=W_{+} ;} \\
& {\left[W_{13}, W_{+}\right]=W_{+} P_{1}+W_{23} P_{+}, \quad\left[W_{13}, W_{-}\right]=-W_{-} P_{1}+W_{23} P_{-},} \\
& {\left[W_{23}, W_{+}\right]=W_{+} P_{2}-W_{13} P_{+}, \quad\left[W_{23}, W_{-}\right]=-W_{-} P_{2}-W_{13} P_{-},} \\
& {\left[W_{23}, W_{13}\right]=W_{+} P_{-}+W_{-} P_{+}, \quad\left[W_{+}, W_{-}\right]=W_{13} P_{1}+W_{23} P_{2} .}
\end{aligned}
$$

The quantum analogues of the above relations, which allow to prove that $W_{q}^{2} 3$. 8) belongs to the center of $U_{z} \mathcal{P}(3+1)$, are:

$$
\begin{aligned}
& {\left[J_{3}, W_{13}^{q}\right] }=-W_{23}^{q}, \quad\left[F_{1}, W_{13}^{q}\right]=z^{2} W_{+}^{q} P_{1} P_{2}, \\
& {\left[E_{2}, W_{13}^{q}\right] }=W_{+}^{q} \cosh z P_{+}, \quad\left[K_{3}, W_{13}^{q}\right]=z W_{+}^{q} P_{2} \sinh z P_{+}, \\
& {\left[F_{2}, W_{13}^{q}\right] }=-W_{-}^{q} \cosh z P_{+}+z^{2} W_{+}^{q}\left\{M_{q}^{2}+P_{2}^{2}\right\} \\
& {\left[J_{3}, W_{23}^{q}\right] }=W_{13}^{q}, \quad\left[F_{2}, W_{23}^{q}\right]=-z^{2} W_{+}^{q} P_{1} P_{2}, \\
& {\left[E_{1}, W_{23}^{q}\right] }=-W_{+}^{q} \cosh z P_{+}, \quad\left[K_{3}, W_{23}^{q}\right]=-z W_{+}^{q} P_{1} \sinh z P_{+}, \\
& {\left[F_{1}, W_{23}^{q}\right] }=W_{-}^{q} \cosh z P_{+}-z^{2} W_{+}^{q}\left\{M_{q}^{2}+P_{1}^{2}\right\} \\
& {\left[E_{1}, W_{-}^{q}\right] }=W_{23}^{q}, \quad\left[F_{1}, W_{-}^{q}\right]=-z^{2} W_{+}^{q} P_{1} P_{-}, \\
& {\left[E_{2}, W_{-}^{q}\right] }=-W_{13}^{q}, \quad\left[F_{2}, W_{-}^{q}\right]=-z^{2} W_{+}^{q} P_{2} P_{-}, \\
& {\left[K_{3}, W_{-}^{q}\right] }=-W_{-}^{q} \cosh z P_{+}+z^{2} W_{+}^{q}\left\{M_{q}^{2}-P_{-} \frac{\sinh z P_{+}}{z}\right\} \\
& {\left[F_{1}, W_{+}^{q}\right]=-W_{23}^{q}, \quad\left[F_{2}, W_{+}^{q}\right]=W_{13}^{q}, \quad\left[K_{3}, W_{+}^{q}\right]=W_{+}^{q} \cosh z P_{+} ; } \\
& {\left[W_{13}^{q}, W_{+}^{q}\right]=W_{+}^{q} P_{1} \cosh z P_{+}+W_{23}^{q} \frac{\sinh z P_{+}}{z}, } \\
& {\left[W_{23}^{q}, W_{+}^{q}\right]=W_{+}^{q} P_{2} \cosh z P_{+}-W_{13}^{q} \frac{\sinh z P_{+}}{z}, } \\
& {\left[W_{23}^{q}, W_{13}^{q}\right]=W_{+}^{q}\left\{P_{-} \cosh z P_{+}-z M_{q}^{2} \sinh z P_{+}\right\}+W_{-}^{q} \frac{\sinh 2 z P_{+}}{2 z} } \\
& {\left[W_{13}^{q}, W_{-}^{q}\right]=-W_{-}^{q} P_{1} \cosh z P_{+}+W_{23}^{q} P_{-} \cosh z P_{+}+z^{2} M_{q}^{2} W_{+}^{q}, } \\
& {\left[W_{+}^{q}, W_{-}^{q}\right]=-W_{-}^{q} P_{2} \cosh z P_{+}-W_{13}^{q} P_{-} \cosh z P_{+}+z^{2} M_{q}^{2} W_{+}^{q}, } \\
& W_{1}^{q}+W_{23}^{q} P_{2} .
\end{aligned}
$$




\section{Acknowledgements}

This work has been partially supported by DGICYT de España (Projects PB910196 and PB92-0255).

\section{References}

[1] P.A.M. Dirac, Rev. Mod. Phys. 21 (1949) 392.

[2] H. Leutwyler and J. Stern, Ann. Phys. (N. Y.) 112 (1978) 94.

[3] I.B. Kogut and L. Susskind, Phys. Reports 8C (1973) 75.

[4] I.B. Kogut and D.E. Soper, Phys. Rev. D1 (1970) 2901.

[5] H. Leutwyler and J. Stern, Phys. Lett. B73 (1978) 75.

[6] H. H. Liu and D.E. Soper, Phys. Rev. D48 (1993) 1841.

[7] J. Lukierski A. Nowicky and H. Ruegg, Phys. Lett. B. 293 (1992) 344.

[8] M. Chiachian and A. Demichev, Phys. Lett. B. 304 (1993) 220.

[9] A. Ballesteros, F.J. Herranz, M.A. del Olmo and M. Santander, J. Math. Phys. 35 (1994) 4928.

[10] A. Ballesteros, F.J. Herranz, M.A. del Olmo and M. Santander, J. Phys. A: Math. Gen. to appear.

[11] A. Ballesteros, F.J. Herranz, M.A. del Olmo and M. Santander, Lett. Math. Phys. to appear.

[12] S. Zakrzewski, Poisson Poincaré Groups, preprint hep-th/9412099.

[13] M. Maggiore, Phys. Rev. D49 (1994) 5182. 